\title{
SUPERPIXEL SEGMENTATION FOR POLSAR IMAGES BASED ON HEXAGON INITIALIZATION AND EDGE REFINEMENT
}

\author{
Meilin $\mathrm{Li}^{1}$, Huanxin Zou*1, Qian $\mathrm{Ma}^{1}$, Jiachi $\mathrm{Sun}^{1}, \mathrm{Xu} \mathrm{Cao}^{1}$, Xianxiang Qin ${ }^{2}$ \\ ${ }^{1}$ College of Electronic Science and Technology, National University of Defense Technology, Changsha, China - \\ summit_mll@qq.com, hxzou2008@163.com,(2233809618,445219733,1135459767)@qq.com \\ ${ }^{2}$ School of Information and Navigation, Air Force Engineering University, Xi’an, China - qinxianxiang@126.com
}

KEY WORDS: PolSAR image, Superpixel segmentation, Hexagon, Unstable pixels, Edge refinement, Initialization

\begin{abstract}
:
Superpixel segmentation for PolSAR images can heavily decrease the number of primitives for subsequent interpretation while reducing the impact of speckle noise. However, traditional superpixel segmentation methods for PolSAR images only focus on the boundary adherence, the significance of superpixel segmentation will be lost when the accuracy is improved at the expense of computation efficiency. To solve this problem, this paper proposes a novel superpixel segmentation algorithm for PolSAR images based on hexagon initialization and edge refinement. First, the PolSAR image is initialized as hexagonal distribution, where the complexity of searching pixels for relabelling in the local regions can be reduced by $30 \%$ theoretically. Second, all pixels in the PolSAR image are initialized as unstable pixels based on the hexagonal superpixels, which can boost the segmentation performance in the heterogeneous regions and effectively maintain all the potential edge pixels. Third, the revised Wishart distance and the spatial distance are integrated as a distance measure to relabel all unstable pixels. Finally, the postprocessing procedure based on a dissimilarity measure is applied to generate the final superpixels. Extensive experiments conducted on both the simulated and realworld PolSAR images demonstrate the superiority and effectiveness of our proposed algorithm in terms of computation efficiency and segmentation accuracy, compared to three other state-of-the-art methods.
\end{abstract}

\section{INTRODUCTION}

Polarimetric synthetic aperture radar (PolSAR) is appealing in virtue of containing richer scatter information and the excellent characteristics of all-day and all-weather operation (Debanshu et al., 2018). PolSAR utilizes vertical and horizontal polarization for alternately transmitting and receiving radar signals to obtain quad-polarization data. Since any target has a specified variable polarization effect on the radiated electromagnetic waves, PolSAR can obtain the abundant scattering information of ground objects, which has broad application prospects in the fields of earth resources, disaster monitoring, urban planning and military reconnaissance. Therefore, the interpretation of PolSAR images has been paid extensive attention. Specifically, object-based interpretation processing has received a considerable amount of concentration for reasons of better noise resistance and lower subsequent primitives (Zou et al., 2019). Furthermore, the superpixel segmentation method can effectively generate the controllable number, regular shape and compact regions, which attracts widespread attention.

Superpixels have been widely used in various aspects, such as target tracking, change detection and classification. Superpixel segmentation methods can be generally categorized into two classes:

1) Graph based methods: the typical algorithms mainly include Normalized Cuts (Ncut) (Ren et al., 2003), Entropy Rate (ER) Superpixels (Liu et al., 2011), and Proposals for Objects from Improved Seeds and Energies (Humayun et al., 2015), etc.

2) Gradient ascent methods: include the classical algorithms of Simple Linear Iterative Clustering (SLIC) (Achanta et al., 2010),
Linear Spectral Clustering (LSC) (Li et al., 2015), Superpixels Extracted via Energy-Driven Sampling (Michaels et al., 2015), etc.

However, all the algorithms mentioned above are proposed for optical images, and the poor performance may be obtained when they are directly applied to PolSAR images. Due to the unique imaging mechanism of the SAR system, there are amount of inherent speckle noise in PolSAR images which makes the regions of same class have severe internal fluctuation. However, optical images are not subject to such noise severely, then it is destined to need the difference between the superpixel generation in PolSAR and optical images. Therefore, some scholars have made improvements in the aforementioned algorithms for PolSAR images.

Improvements on graph based methods: Liu et al. (2013) modified the Ncut algorithm by incorporating the revised Wishart distance and edge map to generate superpixels. However, the boundary adherence of small-sized regions is unsatisfactory due to the limitations of the algorithm framework. Wang et al. (2017) introduced an improved ER into PolSAR images, which take into account the uniformity of PolSAR images by combining Wishart distance and spherically invariant random vector (SIRV) distance. The integrated distance takes advantage of the SIRV model and the Gaussian model, but the operation simplicity is poor, which leads to lower computation efficiency.

Improvements on gradient ascent methods: SLIC is popular because of its good segmentation performance and flexibility. Qin et al. (2015) incorporated the revised Wishart distance into

* Corresponding author 
the distance measure of the SLIC algorithm (POL-SLIC algorithm) with better boundary adherence but poor regularity. Song et al. (2019) utilized hybrid discriminative random field model to improve the distance measurement of SLIC, but didn't attention to the flexibility and time consumption. Considering the importance of 1-D image entropy (Tirandaz et al., 2020), the entropy of the image is calculated and used to determine the number of superpixels based on SLIC. Gadhiya et al. (2020) utilized the Pauli decomposition of a PolSAR image as an input to the SLIC algorithm, and applied the generated superpixels to the classification.

Zhu et al. (2015) introduced a fast superpixel segmentation algorithm by iterative edge refinement (IER), which is fast, homogeneous regular and boundary-adherent for optical images Zhang et al. (2017) replaced the distance of the CIELAB space in the IER algorithm with a revised Wishart distance, which is called POL-IER. Compared with other algorithms, POL-IER can achieve higher segmentation accuracy, and meanwhile, the computation efficiency is also improved to some extent. But there is still no good balance between the accuracy and time consumption. The superpixel segmentation algorithm for PolSAR images is designed to decrease subsequent primitives for interpretation while reducing the impact of speckle noise. As a preprocessing step, lower segmentation accuracy can directly affect the interpretation results. However, the significance of superpixels will be lost as the accuracy is improved at the expense of computation efficiency.

In order to balance the segmentation results and computation efficiency, this paper proposes a novel superpixel segmentation algorithm for PolSAR images based on hexagon initialization. Different from the IER originally designed for the superpixel generation of optical images, the PolSAR image is firstly initialized into multiple regular hexagonal superpixels. Compared with the original square distribution, the number of searchable pixels can be reduced by $30 \%$ in the local searching regions, which greatly reduces the unnecessary time consumption. Next, fully considering the particularity of slim and small-size regions for PolSAR images, all of pixels are initialized as unstable points based on hexagons. This is beneficial for generating superpixels both in the heterogeneous and homogeneous regions and further boosting the boundary adherence. Thirdly, the integrated distance combined with revised Wishart distance and spatial distance is applied to relabel unstable points. Finally, the postprocessing by dissimilarity measure is utilized to obtain the final superpixels. The main contributions of our work are summarized as follows: 1) The hexagon initialization is firstly introduced into the superpixel generation for PolSAR images. 2)The experimental results conducted on both the simulated and real-world PolSAR images effectively demonstrate that our algorithm can provide better balanced trade-offs between the computation efficiency and segmentation accuracy, compared to three other competitive state-of-the-art methods.

\section{METHODOLOGY}

The proposed method contains three modularized steps. First, the PolSAR image is initialized as hexagonal distribution. Then, based on hexagonal distribution, all of pixels are initialized as unstable points for relabelling by integrated distance measure. Finally, the postprocessing is performed to eliminate isolated small regions.

\subsection{Hexagon Initialization}

In an image, unstable pixels (Zhu et al., 2015) are those pixels whose labels are likely to change and should be checked in the next iteration. The definition of unstable pixels is given as follows:

$$
\mathrm{UP}=\{p \mid n t(p) \neq n t(q) \text { and } n t(q) \neq t(q), q \in N b(p)\}
$$

where $p$ and $q$ represent pixels in the image domain. $N b(p)$ is the neighborhood function and a 4-connected neighborhood is utilized in the experiments. $t(i)$ represents the label of $i$ and $n t(i)$ represents the new label after one iteration, $i=p, q$.

Both in IER and POL-IER methods, the images are initialized as square distribution shown as Fig. 1(a). Rectangles in a black solid line are initialized superpixels. Centers $C_{i 0}, C_{i 1}, C_{i 2}, C_{i 3}$, $C_{i 4}, C_{i 5}, C_{i 6}, C_{i 7}$ and $C_{i 8}$ are sampled with an interval of $S$ pixels. Square superpixels display central symmetry, but the regions containing the image edges are generally asymmetric. Considering the boundary adherence of superpixels across image edges, it can better meet the complex terrain condition while the initialized superpixel is a polygon. On the other hand, the symmetry of hexagons is both central symmetry and axial symmetry.

As shown in Fig. 1 (b), there is the hexagonal initialization with the clustering centers $C_{j 0}, C_{j 1}, C_{j 2}, C_{j 3}, C_{j 4}$ and $C_{j 5}$. Hexagons in a black solid line are initialized superpixels, which are sampled horizontally at intervals of $S_{h}$ pixels and $S_{v}$ pixels in the vertical direction. To facilitate subsequent comparisons, set the hexagon superpixel equal to the square superpixel in area, then the side length of the square and hexagon need to satisfy the following geometric relationship:

$$
\frac{S^{2}}{H^{2}}=\frac{3 \sqrt{3}}{2}
$$

According to the geometric characteristics of the hexagonal distribution, the horizontal and vertical distances of adjacent superpixels are:

$$
\begin{aligned}
& S_{h}=\sqrt{3} H \\
& S_{v}=\frac{3}{2} H
\end{aligned}
$$

Combined Eq.(2)-(4), the horizontal distance $S_{h}$ and vertical distance $S_{v}$ can be obtained as follows, respectively:

$$
\begin{aligned}
& S_{h}=\sqrt{\frac{2}{\sqrt{3}}} \cdot S \\
& S_{v}=\sqrt{\frac{\sqrt{3}}{2}} \cdot S
\end{aligned}
$$

Specifically, our proposed method searches the superpixel center for a certain unstable pixel within the size of $2 S \times 2 S$. As for square distribution, there are nine clustering centers $C_{i 0}, C_{i 1}$, $C_{i 2} C_{i 3}, C_{i 4}, C_{i 5}, C_{i 6}, C_{i 7}$ and $C_{i 8}$ in the local regions. Fig. 1(a) depicts that the search range of the unstable pixel $i$ is the rectangle marked by the blue dotted. In consequence, the integrated distance need to be calculated up to nine times for final assignment. When the input pixel of the PolSAR image is $N$, the complexity of each clustering iteration is $9 N$. 


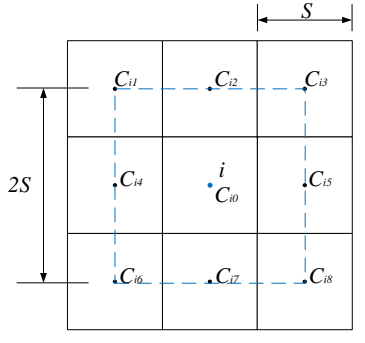

(a)

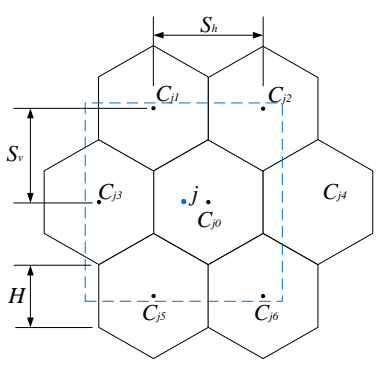

(b)
Figure 1. Distribution of Cluster Centers. (a) Square distribution. (b) Hexagonal distribution.

With regard to hexagonal distribution shown as Fig. 1(b), there are six adjacent superpixels $\left(C_{j 0}, C_{j 1}, C_{j 2}, C_{j 3}, C_{j 4}\right.$ and $\left.C_{j 5}\right)$ in the local regions. This means that, at most, just six distance calculations needed for determining the superpixel which the unstable pixel $j$ belongs. Compared with square distribution, the hexagonal distribution owns a lower complexity of $6 \mathrm{~N}$ for one iteration of the clustering, which significantly improves the computation efficiency. Meanwhile, polygonal distribution can be more efficiently attached to edges for the heterogeneous areas of PolSAR images. At the same time, not any regular polygon can initialize the image completely (close to cover the entire plane), and the time consumption will increase as the number of edges increases. The hexagon initialization can just avoid the aforementioned defects, furthermore, it is negligible for the construction of hexagons compared with the time saved by using hexagon initialization.

\subsection{Initialization of unstable Pixels}

Fig. 2(a) displays that IER method divides the image into squares and initializes the unstable pixels as the square edge pixels with red regions. Due to optical images always contain several regions of regularity and homogeneity, this kind of initialization has slight effect on the boundary adherence of the final superpixels.

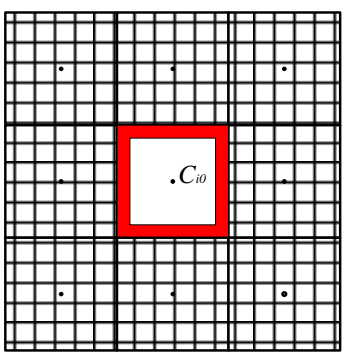

(a)

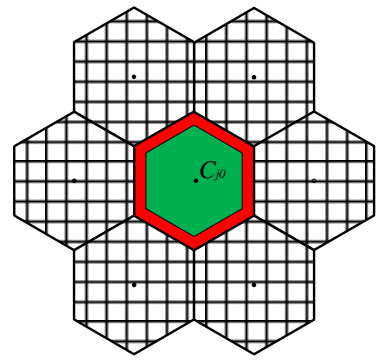

(b)
Figure 2. The sketch map of unstable pixels initialization of two methods. (a) IER method; (b) proposed method.

By contrast, PolSAR images usually include many small regions, slim regions and strong point targets, which can provide important information for image processing. When applied to PolSAR images, this implementation of initialization may provide poor superpixels with losing many potential edges. In addition, the properties of good boundary adherence and homogeneous regularity should be obtained with as few superpixels as possible. However, the final results of IER can be severely affected by the initial square width. When the original square width is large relative to the image size, many real edge pixels may locate in the middle of the square, thus leading to missing some edges. Therefore, with small grid width, IER may work well, and otherwise the opposite, which is not expected.

Therefore, we initialize the unstable pixels as all the pixels based on hexagonal distribution in PolSAR images, instead of the square edges. As Shown in Fig. 2(b), our proposed initial unstable pixels consist of the red pixels, green pixels and the corresponding cluster center filled by black. By doing so, all the potential edges in PolSAR images will be maintained, resulting in accurate boundary adherence and thus accurate superpixels. Although boundary adherence of the POL-IER method has improved, in the face of complex terrain distribution of PolSAR images, it still cannot meet demand. While the proposed method optimizes the code to achieve the accomplishment that all of pixels are initialized to unstable pixels based on the hexagons. Consequently, both in the heterogeneous and homogeneous regions can be segmented availably, resulting in accurate boundary adherence and thus accurate superpixels.

\subsection{Local Relabeling and Postprocessing}

Concerning the inherent speckle noise for PolSAR images, we utilize the revised Wishart distance (Qin et al., 2015) combined with spatial distance. The revised Wishart distance between a pixel $i$ with the coherency matrix $\mathbf{T}_{i}$ and the $j$ th cluster $R_{j}$ with the center coherency matrices $\mathbf{C}_{j}=\left(\sum_{n=1}^{N_{j}} \mathbf{T}_{n}\right) / N_{j}$ is:

$$
d_{R W}\left(i, R_{j}\right)=\operatorname{In}\left(\frac{\left|\mathbf{C}_{j}\right|}{\left|\mathbf{T}_{i}\right|}\right)+\operatorname{Tr}\left(\mathbf{C}_{j}^{-1} \mathbf{T}_{i}\right)-q
$$

The spatial distance is defined by

$$
d_{s}(i, j)=\sqrt{\left(x_{j}-x_{i}\right)^{2}+\left(y_{j}-y_{i}\right)^{2}}
$$

where the subscript $i$ and $j$ represent the cluster center of the $i$ th superpixel and the $j$ th unstable pixel, respectively.

Because the revised Wishart distance will be calculated many times in the local relabeling step, a large amount of time will be cost in the computation. Therefore, a fast implementation (Zhang et al., 2017) of the revised Wishart distance is employed in this paper to further increase the computation efficiency. Let $\mathbf{w}_{j}=f\left(\left(\mathbf{T}_{j}^{-1}\right)^{T}\right)$ and $\mathbf{t}_{i}=f\left(\mathbf{T}_{i}\right)$, where $(.)^{T}$ means a matrix transpose without conjugation, and $f($.$) be a function that$ arranges all the elements of the matrix into a vector. Then it is easy to notice the fact that $\operatorname{Tr}\left(\mathbf{C}_{j}^{-1} \mathbf{T}_{i}\right)=\left(\mathbf{w}_{j}\right)^{T} \mathbf{t}_{i}$, where both $\mathbf{w}_{j}$ and $\mathbf{t}_{i}$ are 9-dim vectors. Contrary to the high-computation of $\mathbf{C}_{j}^{-1} \mathbf{T}$, only 9 multiplication operations and 8 addition operations are needed to calculate $\left(\mathbf{w}_{j}\right)^{T} \mathbf{t}_{i}$, which is only onethird of what $\operatorname{Tr}\left(\mathbf{C}_{j}^{-1} \mathbf{T}_{i}\right)$ needs in traditional way. Therefore, the revised Wishart distance can be represented by

$$
d_{R W}\left(i, R_{j}\right)=\operatorname{In}\left(\frac{\left|\mathbf{C}_{j}\right|}{\left|\mathbf{T}_{i}\right|}\right)+\left(\mathbf{w}_{j}\right)^{T} \mathbf{t}_{i}-q
$$

Then, the integrated distance is shown as follows: 


$$
D\left(i, R_{j}\right)=\left(\frac{d_{R W}\left(i, R_{j}\right)}{m}\right)^{2}+\left(\frac{d_{s}\left(i, R_{j}\right)}{S}\right)^{2}
$$

Where $S$ is the half of the search range width and $m$ is the compactness parameter.

Finally, in order to merge the generated small isolated regions as well as to preserve the strong point targets, a postprocessing procedure based on dissimilarity measure (Zhang et al., 2017) is adopted in this paper. When the size of a superpixel is smaller than $N_{t h}=S^{2} / 4$, the dissimilarities between this superpixel and the superpixels in its 8-neighborhood will be calculated. If the smallest dissimilarity value is smaller than a predefined threshold $G_{t h}$, we merge this superpixel into the neighbor with the smallest dissimilarity between them. If not, move to the next superpixel. The dissimilarity measure between two superpixels $R_{i}$ and $R_{j}$ is defined as follows:

$$
G\left(R_{i}, R_{j}\right)=\frac{1}{q}\left\|\frac{\mathbf{C}_{i}^{\text {diag }}-\mathbf{C}_{j}^{\text {diag }}}{\mathbf{C}_{i}^{\text {diag }}+\mathbf{C}_{j}^{\text {diag }}}\right\|_{1}
$$

where $\mathbf{C}^{\text {diag }}$ means the vector consisting of the diagonal elements of the center coherency matrix of a superpixel and \|\|$_{1}$ denotes 1-norm of a matrix. Since the dissimilarity $G$ belongs to $[0,1], G_{t h}$ is set as 0.3 in all the experiments throughout this paper. The main steps of our algorithm for PolSAR data can be summarized as follow.

Input: The initial width $S$, PolSAR image $I$

Step 1. Initialization

Initialize the image as hexagonal distribution.

Initialize all the pixels as the unstable pixels based on hexagonal superpixels. Set the iteration index iter $=0$.

Step 2. Local relabeling

If iter $\geq$ itermax, or the unstable point set is empty, then the algorithm ends and goes to Step 4. Or relabel all the unstable pixels by the integrated distance defined in Equation (10). Assign each unstable pixel whose searching area is $2 S \times 2 S$ to the closest cluster whose location is covered by the searching area.

Step 3. Updating

Update the superpixel models and the unstable pixel set by the definition (1). Set iter $=i t e r+1$ and return to Step2.

Step 4. Postprocessing

Search the superpixels with the size smaller than $N_{t h}$. If the smallest dissimilarity calculated by Equation (11) is smaller than $G_{\text {th }}$, merge it into the closest adjacent superpixel. If not, move to the next superpixel until each superpixel is checked.

Output: Superpixels

Algorithm 1. Proposed Algorithm

\section{EXPERIMENTS}

To evaluate our proposed method, extensive experiments on two simulated PolSAR images and one real-world image from
AirSAR were conducted. Section 3.1 introduced the comparison experiments of the square distribution and hexagonal distribution, evaluation on the initialization of unstable pixels and comparison experiments on three state-of-the-art algorithms based on the simulated PolSAR data. The experiments demonstrated that the proposed method showed good performance with respect to the four commonly used criterions. In Section 3.2, experiments and discussions on the real-world PolSAR image were contained and the results showed that the proposed method can provide better balanced trade-offs between the computational efficiency and boundary adherence compared with other three methods. All the experiments were performed on the computer with $3.30 \mathrm{GHz}$ Intel Pentium CPU, 64 GB memory and MATLAB Code except for POL-LSC method is implied by MATLAB mixed with C Code.

\subsection{Evaluation on Simulated PolSAR Images}

In our experiments, two simulated PolSAR images with three same regular regions but different sizes were employed. The generation of PolSAR simulated images is based on the inverse transform method (Qin et al., 2015), with $400 \times 400$ pixels and $500 \times 500$ pixels. The simulated image with the size of $400 \times 400$ is shown as Fig. 3(a) and corresponding ground truth is given in Fig. 3(b).

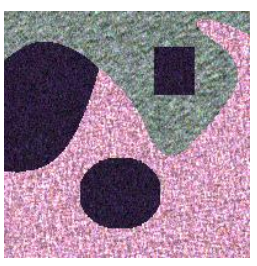

(a)

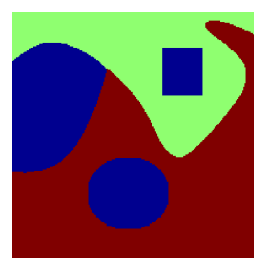

(b)
Figure 3. The simulated image with the size of $400 \times 400$ and corresponding ground truth. (a) Pauli-RGB image of the simulated PolSAR data. (b) The corresponding ground truth.

To evaluate the performance of different methods for superpixels' generation, all the experiments on the PolSAR images mentioned above were assessed on four criterions: Boundary Recall (BR), Under-segmentation Error (USE), Achievable Segmentation Accuracy (ASA) and the running time.

\subsubsection{Evaluation on Hexagon Initialization}

Our proposed method initializes the PolSAR image into hexagons instead of squares. So as to validate the hexagonal distribution, we improved the initialization of POL-IER method to hexagons (POL-IER+ Hexagon) compared with the original square distribution. The compactness parameter $m$ was set as 1.4 (the best) for the two methods. The results on the two criterions are shown in Fig. 4. The abscissa was set as the half of the search range that is the value of $S$, and the corresponding hexagon width can be calculated according to Equation (2).

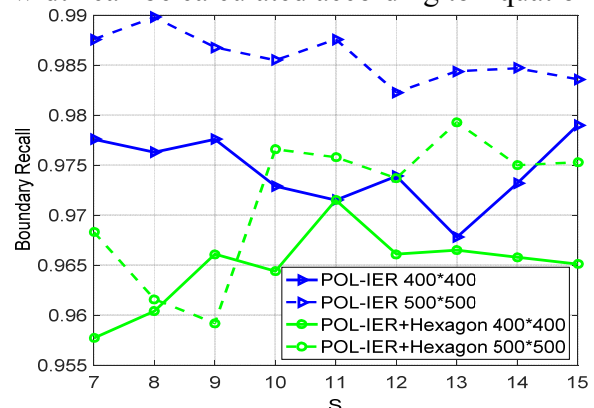

(a) 


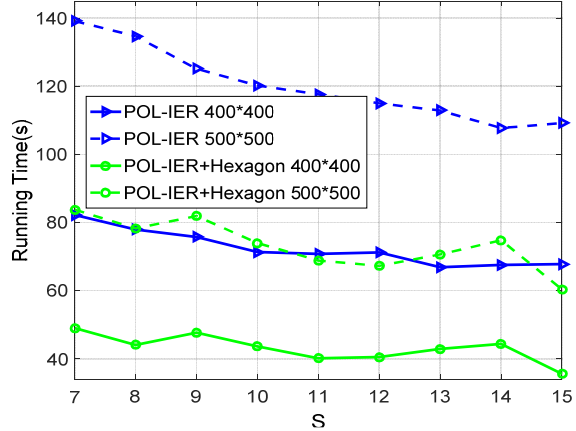

(b)

Figure 4. The comparison of POL-IER with two different distributions, i.e., square distribution and hexagonal distribution with respect to two criterions of (a) BR, (b) Running Time.

We can find that the hexagonal distribution was greatly superior to the square distribution in terms of computation efficiency, as well as the average running time can be significantly reduced by $30 \%$, which is the expected property for superpixel. Fig. 4(b) intuitively shows that the time consumption of hexagonal distribution for the data of $500 \times 500$ is same as the square for the data of $400 \times 400$. This means that hexagon initialization can effectively reduce the number of searchable clustering centers in the same search range which leads to reducing the number of distance calculations. Therefore, the hexagonal distribution is chosen as the initialization in our proposed method. Fig. 4(a) shows that the boundary recall decreased slightly, but the difference is basically about 0.01 . This is owing to that it is faced with the pixels when the image is initialized to hexagons, ideal situation for geometric structure cannot be achieved. In this way, it is more necessary to initialize all the pixels into unstable pixels for the sake of eliminating the above drawback.

\subsubsection{Evaluation on Initialization of Unstable Pixels}

Superpixel segmentation for PolSAR images, as a preprocessing step, must own the good accuracy while maintaining the high computation efficiency. Obviously, it will make the serve impact on subsequent interpretation results with the existence of improper segmentation. IER method only initializes the unstable pixels as the square edge pixels, while the proposed method optimizes the code to achieve the accomplishment that all of pixels are initialized to unstable pixels based on hexagons. As a result, proposed method was compared with the POL-IER to verify the segmentation performance. The number of iterations for proposed method is set to 20 in this paper. It can be clearly seen from Fig. 5 that in the implementation of all pixels are initialized to the unstable pixels, boundary recall is significantly improved, especially when the $S$ is greater than 9 .

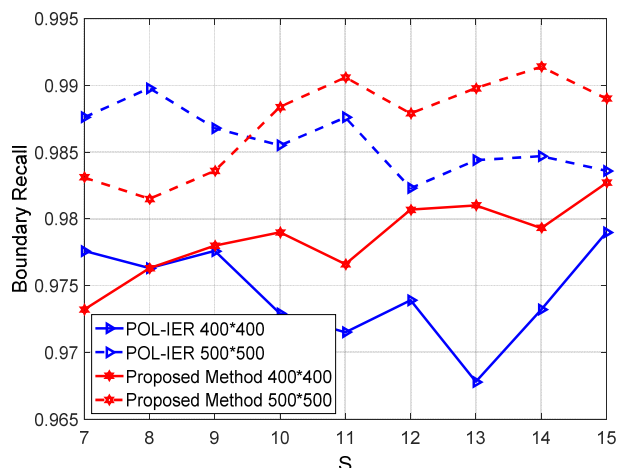

(a)

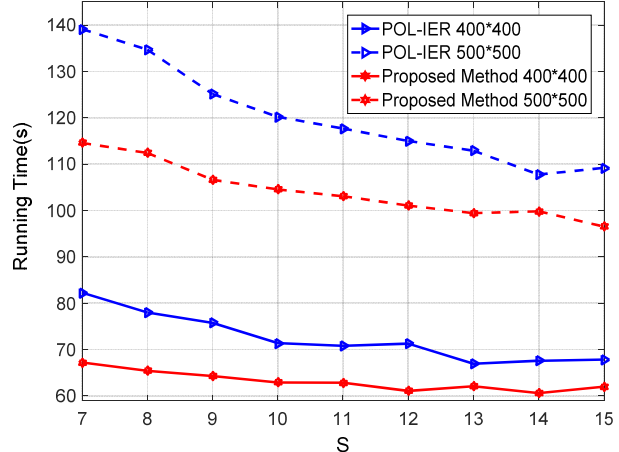

(b)

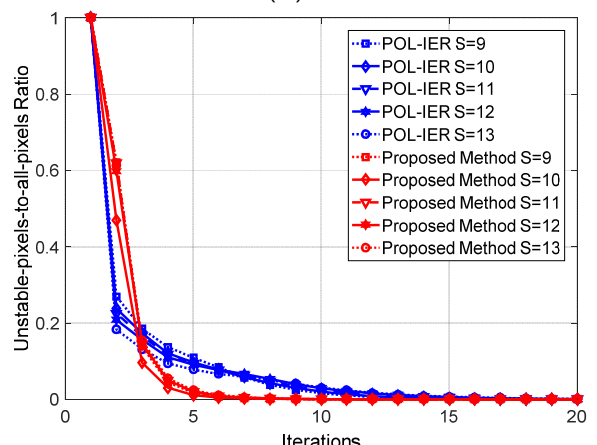

(c)

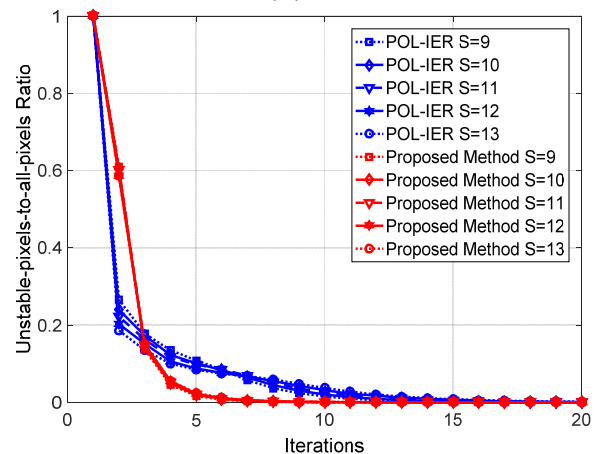

(d)

Figure 5. The comparison of two different initializations of unstable pixels, with respect to four criterions of (a) BR, (b) Running Time, (c) the ratio of unstable pixels to all the pixels in the simulated PolSAR image with size $400 \times 400$ and (d) with size $500 \times 500$.

As can be seen from Fig. 5(e-f), although the number of unstable pixels for the POL-IER method drop sharply in the first few iterations, after three iterations, the falling speed for unstable pixels' number of the proposed method exceeds the POL-IER on account of the superpixels' center of proposed method is placed in the hexagon.

\subsubsection{Evaluation on Four Algorithms}

Four algorithms including POL-SLIC, POL-LSC (Li et al., 2015), POL-IER (Zhang et al., 2017) and proposed method were evaluated on the two simulated images. These four algorithms all belong to gradient ascent methods, which own high computational efficiency so that to fair and objective in comparison. For these methods, the weighting factor of POLLSC was set as 0.2 , the compactness parameter $m$ was set as 1.2 for POL-SLIC, 1.4 for POL-IER and proposed method. Fig. 6 illustrates the results of the four algorithms on two simulated images. For BR, Pol-IER outperforms SLIC-GC, and proposed method is the best of the results, which indicates that the superpixels generated by proposed method have the boundaries 
closest to the real edges among the four methods. POL-LSC method display the worst boundary adherence, the BR of proposed method is about $11 \%$ higher than it when the value of $S$ is 13 with the size $500 \times 500$.

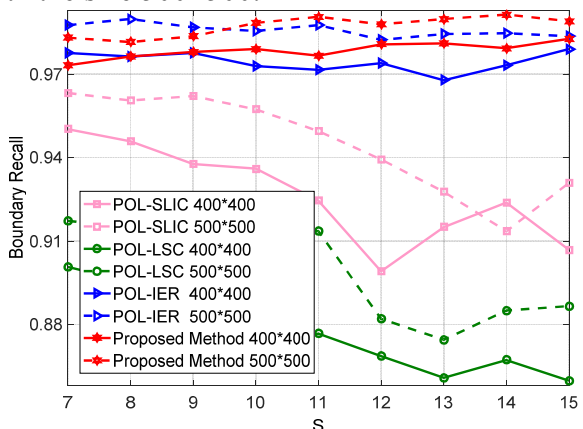

(a)

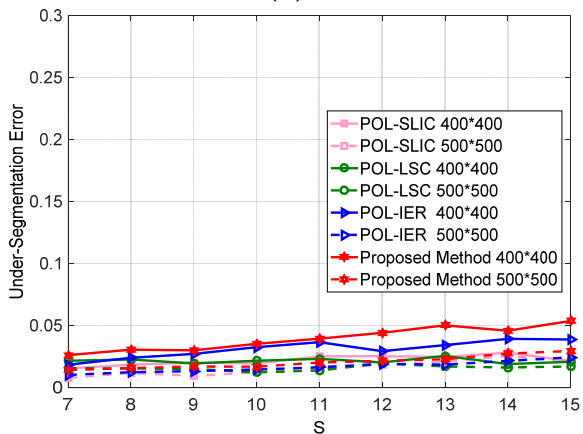

(b)

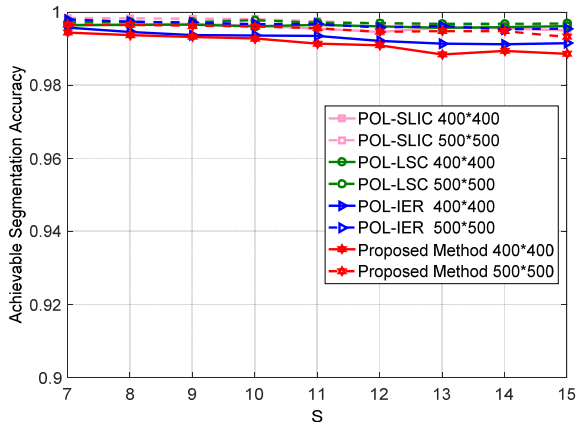

(c)

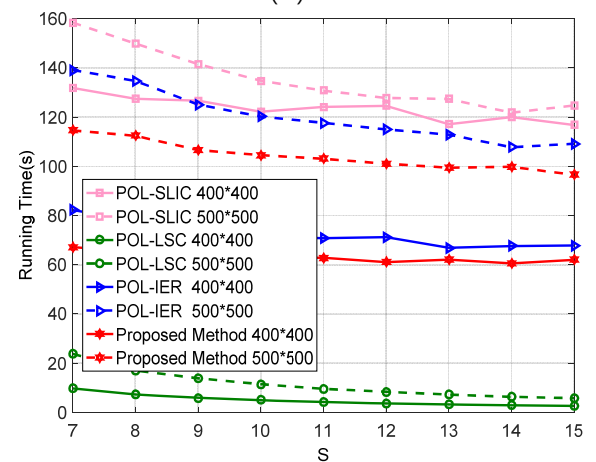

(d)

Figure 6. The results of four algorithms: POL-SLIC, POL-LSC, POL-IER and proposed method. (a) BR. (b) USE. (c) ASA. (d) Running Time.

On the contrary, the BR of the POL-SLIC and POL-LSC methods for simulated images is very low which can occur the more terrible results for the real world images. Apparently, it gives rise to the poor performance on subsequent interpretation steps, which the significance of superpixel segmentation is lost. As shown in Fig. 6(b-c), there is no big difference about USE

and ASA of four methods. On account of that the POL-LSC method was applied by MATLAB mixed with $\mathrm{C}$ Code, the requirement of time costs is lower, but the significance is also lost with the terrible boundary adherence. The running time of Fig. 6(d) once again illustrates that the proposed method maintains the higher computation efficiency along with greater boundary adherence.

\subsection{Evaluation on Real-world PolSAR Images}

The real-world data set is a 4-look AirSAR L-Band PolSAR image with size $750 \times 1024$ from Flevoland, the Netherlands, and the Pauli color-coded image is shown in Fig. 7. The POLSLIC, POL-LSC, POL-IER and proposed method were performed on the real-world image for comparison. The initial width is generally set according to the complexity of terrains empirically. In the experiments, all of the initial widths were set as $S=6$. For these methods, the weighting factor of POL-LSC was set as 0.3 , the compactness parameter $m$ was set as 0.1 for POL-SLIC, 0.4 for POL-IER and proposed method. Fig. 8 shows the generated superpixels of four methods. The first column denotes the final superpixel maps of different methods as well as the red lines superimposed onto the Pauli images for depicting the superpixel boundaries.

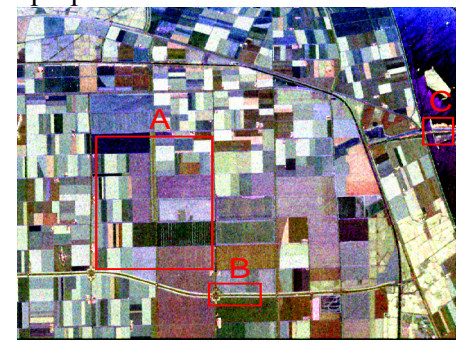

Figure 7. The Pauli-RGB image of the real-world PolSAR data.

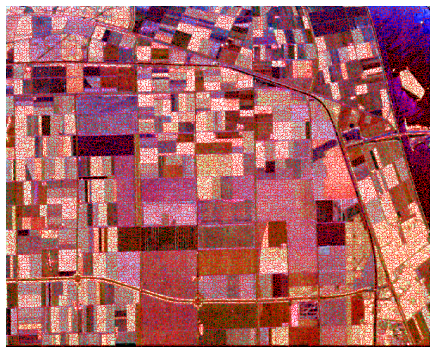

(a)

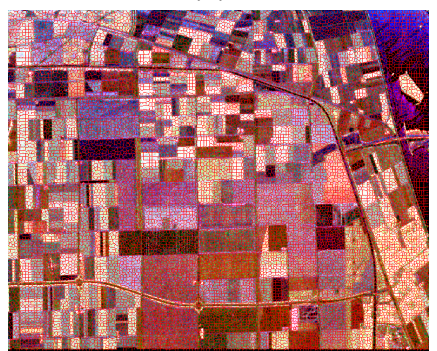

(c)

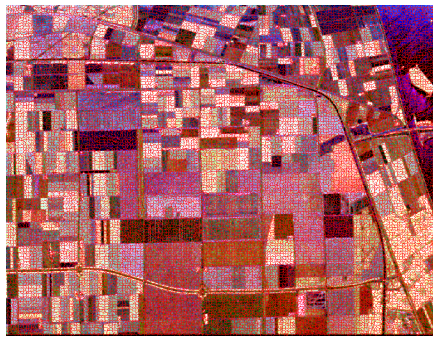

(e)

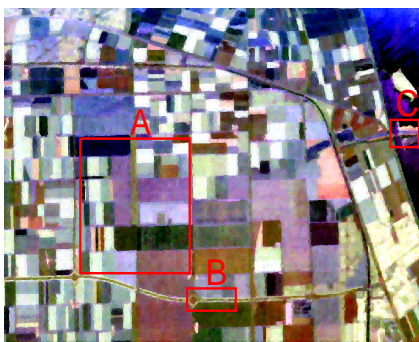

(b)

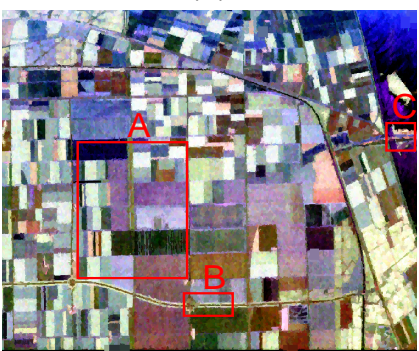

(d)

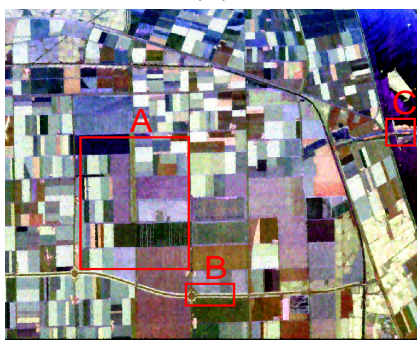

(f) 


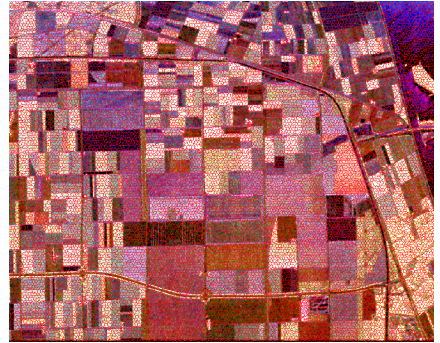

(g)

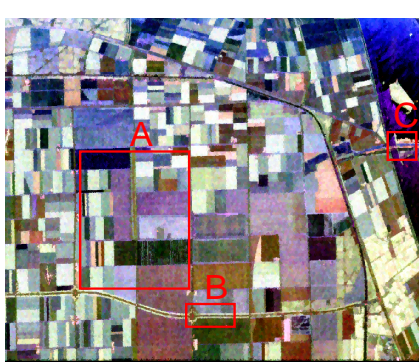

(h)
Figure 8 . The generated superpixels of the four competitive methods. (a)-(b) are the results of POL-SLIC, (c)-(d) are the results of POL-LSC, (e)-(f) are the results of POL-IER and (g)(h) are the results of proposed method.

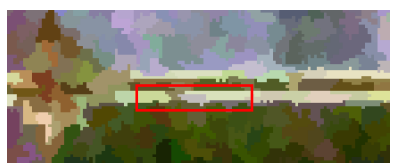

(a)

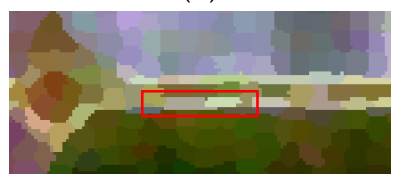

(c)

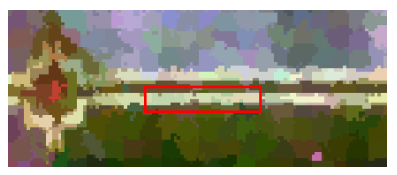

(e)

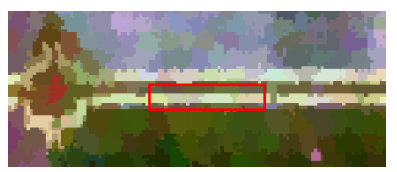

$(\mathrm{g})$

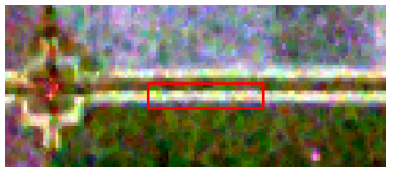

(i)

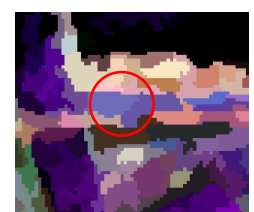

(b)

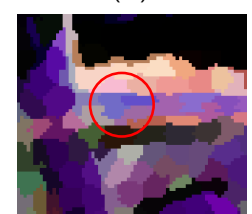

(d)

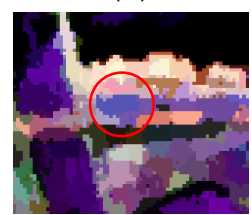

(f)

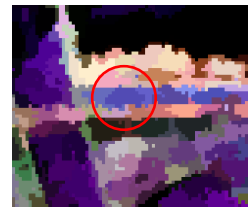

(h)

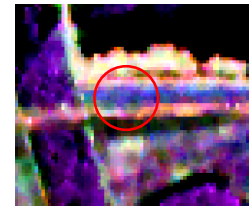

(j)
Figure 9. Superpixels results by POL-SLIC (first line), POLLSC (second line), POL-IER (third line), proposed method (fifth line) and two image patches B and C (fifth line) of Fig. 7.

The second column gives the representation maps, where the coherency matrix of each pixel is replaced by the average value of the superpixel this pixel belongs to. To further evaluate the performance of proposed method, extensive experiments and discussions were conducted on the real-world PolSAR image. It can be seen from Fig. 8 that proposed method performs best with the smoothest boundary of superpixels. For visual clarity, the small regions $\mathrm{B}$ and $\mathrm{C}$ in Fig. 8(b, d, f, h) are enlarged and shown in Fig. 9. The results of Fig. 9(a-b, c-d) shows that POLLSC and POL-SLIC cannot preserve the boundaries of slim regions with irregular generated superpixels, such as the road.
On the one hand, it will make the serve impact on subsequent interpretation results with the existence of improper segmentation. POL-IER and proposed method show the better performance with respect to boundary adherence than other methods.

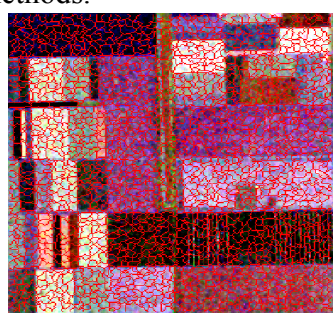

(a)

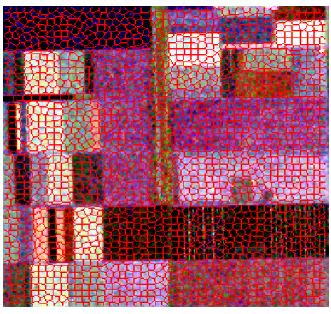

(c)

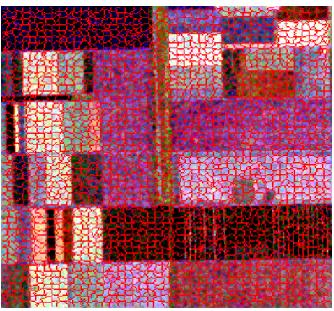

(e)

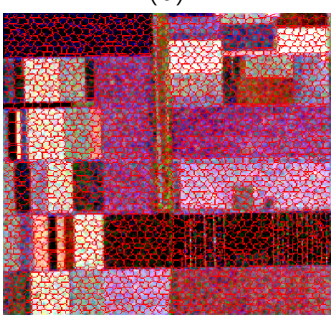

(g)

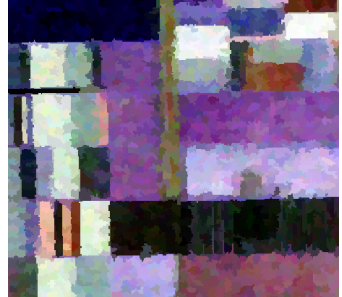

(b)

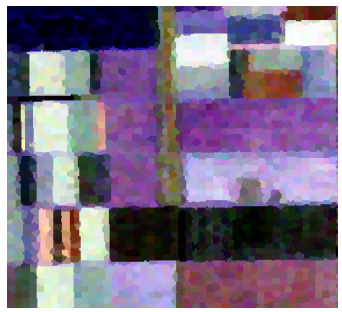

(d)

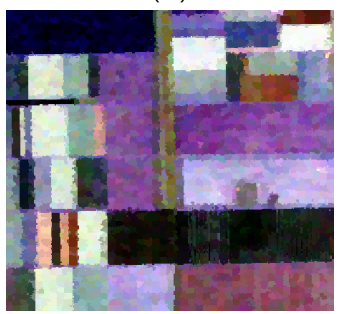

(f)

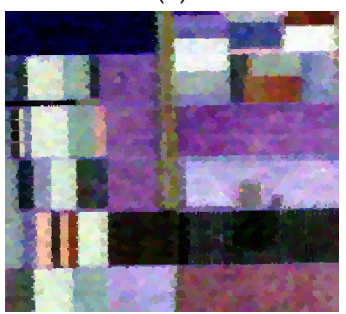

(h)
Figure 10. The enlarged superpixels for region $\mathrm{A}$ of the four competitive methods. (a)-(b) are the results of POL-SLIC, (c)(d) are the results of POL-LSC, (e)-(f) are the results of POL-

IER and $(\mathrm{g})-(\mathrm{h})$ are the results of proposed method.

The performance of these four competitive algorithms on the real-world PolSAR image was evaluated by the visual observation and quantitative evaluation, which the region A by the red rectangle was selected to evaluate quantitatively. Moreover, it is not difficult to find that both in the heterogeneous and homogeneous regions, the results of proposed method are more detailed by comparing Fig. 9(e-g, fh).

\begin{tabular}{cccc}
\hline Algorithm & Clustering & Postprocessing & Total \\
\hline POL-SLIC & 839 & 124 & 963 \\
POL-LSC & 371 & - & 371 \\
POL-IER & 641 & 65 & 706 \\
Proposed Method & 556 & 64 & 620 \\
\hline
\end{tabular}

Table 1. Running Time(s) of four methods for the real-world PolSAR image. 


\begin{tabular}{ccccc}
\hline Algorithm & BR & USE & ASA & Time(s) \\
\hline POL-SLIC & 0.6958 & 0.2558 & 0.9600 & 58 \\
POL-LSC & 0.5872 & 0.2276 & 0.9593 & 5 \\
POL-IER & 0.7762 & 0.2268 & 0.9602 & 32 \\
Proposed Method & 0.7880 & 0.2165 & 0.9607 & 29 \\
\hline
\end{tabular}

Table 2. Four criteria of four methods for the region A of the real-world PolSAR image.

Especially the regions marked with the red rectangle, which demonstrates the superiority of our proposed method in boundary adherence. Furthermore, the running time of proposed method is $12 \%$ faster than the POL-IER by Table 1 , with the better preservation on boundaries of ground terrains. The generated superpixels of region A is shown as Fig. 10, Table 2 displays the corresponding quantitative evaluation. Intuitively, proposed method obtain the highest boundary recall, which is 0.2008 higher than the POL-LSC method, and further verifies the hexagon initialization is more suitable for the complex terrain situation of PolSAR images. Similarly, computation efficiency for proposed method gets the improvement of $9 \%$ than the POL-IER based on the excellent boundary adherence, which validates the effectiveness of hexagon initialization as well as better balanced trade-offs between the computation efficiency and segmentation accuracy.

\section{CONCLUSION}

Most of superpixel segmentation algorithms for optical images are basically perform well, however, without considering the inherent speckle noise for PolSAR images. Although more and more scholars improve the methods for the characters of PolSAR images, there is also a greater need for time consumption with dissatisfactory results of slim regions. For above, a fast superpixel segmentation framework based on the hexagon initialization for PolSAR images is proposed in this paper. To enhance the computation efficiency and decrease the number of distance calculation in the local clustering, proposed method initializes the cluster center into the hexagons which engenders the reduction of searchable pixels. Then, all of pixels were initialized unstable pixels for relabelling by utilizing the integrated distance, fully considering the impact of slim or small regions as well as complex terrain distribution for PolSAR images. Finally, the postprocessing based on dissimilarity measure is adopted to obtain the final superpixels. The experiments conducted on simulated and real-world PolSAR images show that proposed method outperforms the other three competitive methods in boundary adherence, regularity and strong point targets' preservation, especially the time consumption. Although the running time is longer than POL-LSC, it is quite short compared with other methods of superpixel generation for PolSAR images. Furthermore, the significance of superpixel segmentation will be lost with the terrible boundary adherence, such as POL-LSC.

\section{REFERENCES}

Achanta, Radhakrishna, Appu Shaji, Kevin Smith, Aurelien Lucchi, Pascal Fua, and Sabine Susstrunk. 2012: SLIC Superpixels Compared to State-Of-The-Art Superpixel Methods. IEEE Transactions on Pattern Analysis and Machine Intelligence 34(11), 2274-82.

Bergh, M. V. D., Boix, X., Roig, G., Capitani, B. D., and Gool, L. V., 2012: SEEDS: Superpixels Extracted via Energy-Driven Sampling. European Conference on Computer Vision, Springer, Berlin, Heidelberg.
Huanxin Zou, Meilin Li, et al, 2019: Superpixel-Oriented Unsupervised Classification for Polarimetric SAR Imagery Based on Consensus Similarity Network Fusion. IEEE ACCESS 7, 78347-78366.

Humayun, Ahmad, F. Li, and J. M. Rehg, 2015: The Middle Child Problem: Revisiting Parametric Min-Cut and Seeds for Object Proposals. IEEE International Conference on Computer Vision, Santiago, Chile.

Liu, Bin, Hao Hu, Huanyu Wang, Kaizhi Wang, Xingzhao Liu, and Wenxian $\mathrm{Yu}, 2013$ : Superpixel-Based Classification with an Adaptive Number of Classes for Polarimetric SAR Images. IEEE Transactions on Geoscience and Remote Sensing 51 (2), 907-24.

Liu M Y, Tuzel O, Ramalingam S, and R. Chellappa, 2011: Entropy Rate Superpixel Segmentation. IEEE Conference on Computer Vision and Pattern Recognition, Colorado, USA.

Li Z, Chen J, 2015: Superpixel Segmentation Using Linear Spectral Clustering. IEEE Conference on Computer Vision and Pattern Recognition, Boston, USA.

Qin, Fachao, J. Guo, and F. Lang, 2015: Superpixel Segmentation for Polarimetric SAR Imagery Using Local Iterative Clustering. IEEE Geoscience and Remote Sensing Letters 12(1), 13-17.

Ratha, Debanshu, A. Bhattacharya, and A. C. Frery, 2018: Unsupervised Classification of PolSAR Data Using a Scattering Similarity Measure Derived from a Geodesic Distance. IEEE Geoscience and Remote Sensing Letters 15(1), 151-155.

Ren, X., and J. Malik, 2003: Learning A Classification Model for Segmentation. IEEE International Conference on Computer Vision, Nice, France.

Song W, Ming L, Peng Z, Yan Wu and Xiaofeng Tan, 2019: Superpixel-Based Hybrid Discriminative Random Field for Fast PolSAR Image Classification. IEEE ACCESS 7, 24547-24558.

Song Zhu, Danhua Cao, Shixiong Jiang, Yubin Wu, Pan $\mathrm{Hu}$, 2015: Fast Superpixel Segmentation by Iterative Edge Refinement. Electronics Letters 51(3), 230-232.

Tushar Gadhiya and Anil K. Roy, 2020: Superpixel-Driven Optimized Wishart Network for Fast PolSAR Image Classification Using Global k-Means Algorithm. IEEE Transactions on Geoscience and Remote Sensing 58(1), 97-109.

Wang, W., Xiang, D., Ban, Y., Zhang, J., and Wan, J., 2017: Superpixel Segmentation of Polarimetric SAR Images Based on Integrated Distance Measure and Entropy Rate Method. IEEE Journal of Selected Topics in Applied Earth Observations and Remote Sensing 10(9), 1-14.

X., Qin, H. Zou, S. Zhou, and K. Ji, 2015: Simulation of spatially correlated PolSAR images using inverse transform method. Journal of Applied Remote Sensing 9(1), 095082.

Yue, Z., Huanxin, Z., Tiancheng, L., Xianxiang, Q., Shilin, Z., and Kefeng, J, 2016: A Fast Superpixel Segmentation Algorithm for PolSAR Images Based on Edge Refinement and Revised Wishart Distance. Sensors 16(10), 1687.

Zeinab Tirandaz, Gholamreza Akbarizadeh and Hooman Kaabi, 2020: PolSAR image segmentation based on feature extraction and data compression using Weighted Neighborhood Filter Bank and Hidden Markov random field-expectation maximization. MEASUREMENT 153, 107432. 